\title{
Transcatheter Arterial Steroid Injection Therapy Improves the Prognosis of Patients with Acute Liver Failure
}

\section{Akifumi Kuwano}

Kyushu University

\section{Tasuku Okui}

Kyushu University Hospital

Motoyuki Kohjima ( $\nabla$ kohjima@med.kyushu-u.ac.jp )

Kyushu University

\section{Miho Kurokawa}

Kyushu University

\section{Takeshi Goya}

Kyushu University

Masatake Tanaka

Kyushu University

Tomomi Aoyagi

Kyushu University

Motoi Takahashi

Kyushu University

Koji Imoto

Kyushu University

\section{Shigeki Tashiro}

Kyushu University

Hideo Suzuki

Kyushu University

Nobuhiro Fujita

Kyushu University

\section{Yasuhiro Ushijima}

Kyushu University

Kousei Ishigami

Kyushu University

\section{Shoji Tokunaga}

Kyushu University Hospital

\section{Masaki Kato}


Kyushu University

Yoshihiro Ogawa

Kyushu University

\section{Research Article}

Keywords: Acute liver failure, transcatheter arterial steroid injection therapy, intrahepatic microcirculatory disturbance

Posted Date: February 2nd, 2022

DOI: https://doi.org/10.21203/rs.3.rs-1275025/v1

License: (9) This work is licensed under a Creative Commons Attribution 4.0 International License. Read Full License 


\section{Abstract}

\section{Background}

Acute liver failure (ALF) is a disorder defined by coagulopathy and encephalopathy with a poor prognosis. No effective therapies have been established except for liver transplantation (LT). We previously reported a subgroup of patients with acute liver injury who developed microcirculatory disturbance leading to intrahepatic hypoxia. This subgroup can be identified by a low serum ALT/LDH ratio that reflects intrahepatic hypoxia. We also established and reported transcatheter arterial steroid injection therapy (TASIT) as a new treatment of ALF. Here, we analyze the effectiveness of TASIT in a larger cohort and evaluate the impact on ALF patients with or without microcirculatory disturbance.

\section{Methods}

We conducted a single-center retrospective study to evaluate the effectiveness of TASIT in patients with ALF admitted at Kyushu University Hospital between January 2005 and March 2018. TASIT is performed by injecting methylprednisolone via the proper hepatic artery over $1 \mathrm{~h}$ for 3 days. One hundred ninety-four patients with ALF were enrolled in this study. Using a serum ALT/LDH ratio of 1.5 as a cut-off value, we stratified patients with ALF into high-LDH and low-LDH subgroups.

\section{Results}

Of the 87 patients given TASIT, 71 (81.6\%) recovered without any complications and $16(18.4 \%)$ died or underwent LT. Of the 107 patients not administered TASIT, 77 (72.0\%) recovered and $30(28.0 \%)$ progressed to irreversible liver failure. In the high-LDH subgroup, $52(86.7 \%)$ of the 60 patients with TASIT recovered, and the survival rate was significantly higher than that in patients who did not receive TASIT. Multivariate regression analysis revealed that the TASIT procedure was one of the significant prognostic factors in the high-LDH subgroup and was significantly associated with PT\% improvement.

\section{Conclusions}

TASIT is an effective treatment for patients with ALF, especially in those with microcirculatory disturbance.

\section{Background}

Acute liver failure (ALF) is a life-threatening systemic disorder characterized by severe coagulopathy and encephalopathy $[1,2]$. Currently, liver transplantation (LT) is the only treatment proven to improve the prognosis of patients with ALF. There are few established therapeutic procedures because the pathogenesis of ALF is poorly understood [3, 4]. Several recent studies indicate that activated hepatic macrophages might play a key role in acute liver injury [5-7]. Therefore, suppressing intrahepatic macrophage activity could be a useful treatment. Corticosteroids are known to inactivate macrophages and have been used to treat ALI (acute liver injury) [8, 9], but their effectiveness has not been confirmed 
[10-13]. We speculated that corticosteroid administration via a peripheral vein might not achieve sufficient concentration in the liver due to the hepatic microcirculatory disturbance in ALF. To overcome this, we established a novel treatment using transcatheter arterial steroid injection therapy (TASIT) for patients with ALI. We reported its efficacy and safety in 2006 [14]. Here, we analyze the effectiveness of TASIT for ALI treatment in a larger cohort.

Hepatic microcirculatory disturbance and subsequent intrahepatic hypoxia are thought to be involved in the pathogenesis of ALI [15]. The characteristic pathological findings of ALI include massive liver cell necrosis with scarce regeneration. Its clinical features include sudden onset and aggressive expansion of liver damage suggesting blood perfusion disorders. Sinusoidal fibrin deposition with deranged coagulative systems occurs in patients with ALF and experimental animals, which suggests sinusoidal hypercoagulation and microcirculatory disturbance in the pathogenesis of ALF [16-19]. We have previously reported that at least some ALI patients develop microcirculatory disturbance and subsequent intrahepatic hypoxia. A serum ALT/LDH ratio $\leq 1.5$ reflects intrahepatic hypoxia and correlates with the etiology and clinical manifestations of ALI [20]. However, it is not clear whether the pathogenesis might affect the therapeutic response to TASIT.

In this study, we aimed to evaluate the efficacy of TASIT in a larger cohort of patients with ALF and investigate the use of the ALT/LDH ratio in identifying appropriate patients for TASIT.

\section{Methods}

\section{Patients}

We conducted a single-center retrospective study to evaluate the effectiveness of TASIT in patients with ALF admitted at Kyushu University Hospital between January 2005 and March 2018. ALF was diagnosed using criteria established by the Intractable Hepato-Biliary Diseases Study Group in Japan. Patients with previously normal liver function who have prothrombin activity percentage of $40 \%$ or less of the standardized values or international normalized ratios of 1.5 or more caused by severe liver damage within 8 weeks of the onset of the symptoms are diagnosed as ALF, and they are further classified into ALF with or without hepatic coma. In the former, hepatic encephalopathy grade II or more severe hepatic coma develops within 8 weeks [21]. When obvious liver atrophy and hepatic coma were observed on admission, the patient was immediately prepared for LT and excluded from the study. TASIT is performed by injecting methylprednisolone via the proper hepatic artery over $1 \mathrm{~h} \mathrm{(1000} \mathrm{mg/day} \mathrm{for} 3$ days). TASIT was performed after obtaining written informed consent from the patient or, in cases of severe encephalopathy ( $\geq$ grade II), from the patient's family. When consent was not obtained, traditional supportive treatments were given. Plasma exchange was repeated to keep PT\% over 40\% or PT-INR lower than 1.5, and hemodiafiltration was performed if necessary [22]. LT was implemented regardless of the treatment method when liver insufficiency progressed and an appropriate donor was available. Patients with malignant tumors and liver cirrhosis, and patients with late-onset hepatic failure, who have a relatively slow onset of hepatic coma (8-24 weeks), were excluded. 
For patients treated by systemic steroid pulse therapy (SPT), $1000 \mathrm{mg}$ of methylprednisolone was administered intravenously for $1 \mathrm{~h}$ per day for 3 days. In total, 194 patients were enrolled in this study. The etiologies of liver injury were the following: hepatitis A virus (HAV; $n=23)$, hepatitis $B$ virus (HBV; $n=$ 53), autoimmune hepatitis ( $A l H ; n=18)$, drug-induced liver injury (DILI) $(n=4)$, unknown (UK) $(n=65)$, and others $(n=31)$. We evaluated the prognosis (survival, death, or LT), the serum ALT levels (on admission, day 3, and day 7), and PT\% (on admission, day 3, and day 7). Using a serum ALT/LDH ratio of 1.5 as a cut-off value, we stratified all population with ALF into high-LDH and low-LDH subgroups. This study was performed in accordance with the Declaration of Helsinki and approved by Kyushu University Hospital Ethics Committee (No. 27-377 and 28-432). The datasets analyzed during the current study are available in the repository of Department of Medicine and Bioregulatory Science, Graduate School of Medical Sciences, Kyushu University..

\section{Alt/ldh Ratio}

Serum ALT and LDH levels were measured using the 7500 Clinical Analyzer (Hitachi High-Technologies Corporation, Tokyo, Japan). LDH was assayed using an enzymatic rate method with lactate as a substrate. ALT assay was performed without pyridoxal phosphate supplementation. The normal ranges of ALT and LDH in this facility were 6-30 and 119-229 U/L, respectively. To focus on the degree of elevation, we set up an index calculated by the following formula: ALT/LDH ratio $=$ (serum ALT-ULN)/(serum LDH-ULN) (ULN: upper limit of normal) as described previously [20].

\section{Arterial Steroid Injection Protocol}

A 5-French catheter was inserted into the common hepatic artery via the right femoral artery. Before steroid injection, angiography was performed to detect any anomalies of the hepatic artery. If no replaced hepatic artery was found, the tip of the catheter was set at the proper hepatic artery. If there were two hepatic arteries, the catheter was positioned in the branch that showed the widest feeding area. After insertion, $1000 \mathrm{mg}$ of methylprednisolone was infused over $1 \mathrm{~h}$ each day for $3 \mathrm{~d}$, and the catheter was removed immediately after injection on the third day. If a bleeding tendency was observed during this procedure, a once-daily plasma exchange was added to the regimen.

\section{Statistical analysis}

Data were analyzed using the SAS software package (version 9.1.3; SAS Institute, Inc., Cary, NC, USA) and R3.6.3 (https://www.r-project.org/). The results were expressed as mean and standard deviation, or median and interquartile range. Significant differences between groups were assessed using the unpaired Student's $t$-test, Fisher's exact test or $\chi^{2}$-test, Mann-Whitney $U$ test, or the paired-samples t-test. The effects of TASIT on the prognosis of ALF, prothrombin time, and serum ALT value were analyzed using regression models. TASIT, anticoagulation, coma, sex, and age were used as explanatory variables. The factors influencing the prognosis of ALF were investigated by logistic regression analysis, and increases 
in prothrombin time and changes in serum ALT value were analyzed by linear regression analysis. Values of ALT were log-transformed when applying the regression models because the distribution of ALT values was right-skewed. Regression analyses were conducted for high-LDH and low-LDH subgroups, in addition to all population. A p-value $<0.05$ was considered statistically significant, and a p-value $<0.10$ was considered marginally significant.

\section{Results}

\section{Subject characteristics}

Among 194 patients with ALF, 87 patients underwent TASIT (TASIT group) and 107 patients did not (no TASIT group). There were no appreciable adverse effects related to TASIT. Substantial numbers of patients with HAV or of unknown etiology underwent TASIT. On the other hand, HBV and AIH patients were in the majority in the no TASIT group. The TASIT group presented more severe states of ALF than the no TASIT group did. Serum AST, ALT, LDH, and ferritin were significantly higher in the TASIT group. Moreover, the TASIT group showed significantly lower PT\% and higher FDP compared with the no TASIT group. It might further support the severity in TASIT group patients that the proportion of them who underwent plasma exchange and required anticoagulant therapy was higher (Table 1). Microcirculatory disturbance and consequent massive hypoxic hepatocyte damage occurred in some patients with ALF. As we had previously shown that the serum ALT/LDH ratio could identify these patients [20], we stratified them into a high-LDH subgroup (ALT/LDH ratio $\leq 1.5$ ) with hepatic microcirculatory disturbance and a low-LDH subgroup (ALT/LDH ratio > 1.5) (Table 1). The TASIT group had significantly higher serum AST, ALT, LDH, and ferritin than the no TASIT group, and this was also the case in both LDH subgroups and the all population. The differences in PT\% and FDP values between the TASIT group and the no TASIT group observed in the all population were not obvious after stratification into the subgroups.

\section{TASIT improved the prognosis of patients with ALF in the high-LDH subgroup}

Of the 87 patients who received TASIT, 71 (81.6\%) recovered without any complications and 16 patients (18.4\%) died or underwent LT. Of the 107 patients who did not receive TASIT, 77 patients $(72.0 \%)$ survived and $30(28.0 \%)$ progressed to irreversible liver failure. In the high-LDH subgroup, the survival rate of the TASIT group (86.7\%) was significantly higher than that of the no TASIT group $(62.1 \%)(p=0.0079)$. TASIT was not significantly effective in the low-LDH subgroup $(p=0.59)$ (Figure 1$)$. We analyzed the effect of TASIT using regression models instead of propensity score matching considering a large variation in the patient background and the number of each case. Multiple logistic regression analyses showed that the existence of coma was the most significant prognostic factor for survival in the all population $(p<0.001)$, high-LDH subgroup $(p<0.001)$, and low-LDH subgroup $(p<0.001)$. TASIT was the next most significant prognostic factor in the high-LDH subgroup $(p=0.02)$, whereas sex $(p=0.02)$ and age $(p=0.03)$, and not TASIT ( $p=0.39)$, were significant prognostic factors in the low-LDH subgroup (Table 2). 


\section{Tasit Was Involved In The Improvement Of Pt\%}

PT\% is a sensitive marker of hepatic synthetic ability and is used to determine the severity of coagulopathy in patients with ALF [23]. It is also incorporated in most of the prognostic models used worldwide for making major therapeutic decisions, such as LT in acute liver failure or steroid therapy in alcoholic hepatitis [24]. TASIT drastically increased PT\% (TASIT group vs. no TASIT group: $30.06 \% \pm$ $13.04 \%$ on admission, $64.73 \% \pm 20.10 \%$ on day 3 , and $84.09 \% \pm 24.21 \%$ on day 7 vs. $34.66 \% \pm 13.53 \%$ on admission, $54.77 \% \pm 17.63 \%$ on day 3 , and $64.89 \% \pm 21.36 \%$ on day $7, p=0.002$ ), especially in the highLDH subgroup (TASIT group vs. no TASIT group: $30.03 \% \pm 13.33 \%$ on admission, $66.55 \% \pm 22.00 \%$ on day 3 , and $87.56 \% \pm 25.28 \%$ on day 7 vs. $32.71 \% \pm 12.28 \%$ on admission, $49.74 \% \pm 21.42 \%$ on day 3 , and $61.88 \% \pm 21.96 \%$ on day $7, p=0.014$ ), but not in the low-LDH subgroup (Figure 2). By multiple logistic regression analysis, TASIT $(p<0.001)$ and the existence of coma $(p<0.001)$ were significantly associated with the change in PT\% in the all population (Table 2). TASIT could be involved in improving PT\% in the high-LDH subgroup, although with marginal significance $(p=0.07$, Table 2$)$.

\section{TASIT had a limited impact on the improvement of serum ALT levels}

Serum ALT level is a surrogate marker for hepatocyte damage because it is markedly elevated during hepatocyte destruction $[25,26]$. Serum ALT levels were drastically decreased after TASIT (TASIT group vs. no TASIT group: $5102.0 \pm 3129.9 \mathrm{IU} / \mathrm{mL}$ on admission, $813.5 \pm 490.2 \mathrm{IU} / \mathrm{mL}$ on day 3 , and $483.4 \pm 480.5$ $\mathrm{IU} / \mathrm{mL}$ on day 7 vs. $2149.8 \pm 2091.5 \mathrm{IU} / \mathrm{mL}$ on admission, $498.9 \pm 482.9 \mathrm{IU} / \mathrm{mL}$ on day 3 , and $244.7 \pm$ $210.5 \mathrm{IU} / \mathrm{mL}$ on day 7), and we could not find statistical significance due to an inevitable gap of ALT between the two groups (Figure 3). The serum ALT levels in the high- and low-LDH subgroups showed a similar trend. Anticoagulant therapy also decreased the ALT levels (with anticoagulant therapy vs. without anticoagulant therapy: $4240.3 \pm 2998.7 \mathrm{IU} / \mathrm{mL}$ on admission, $669.0 \pm 494.9 \mathrm{IU} / \mathrm{mL}$ on day 3 , and $355.3 \pm$ $349.3 \mathrm{IU} / \mathrm{mL}$ on day 7 vs. $1879.9 \pm 2265.9 \mathrm{IU} / \mathrm{mL}$ on admission, $546.2 \pm 537.9 \mathrm{IU} / \mathrm{mL}$ on day 3 , and 339.9 $\pm 444.5 \mathrm{lU} / \mathrm{mL}$ on day 7$)$. Anticoagulant therapy was the only significant factor related to ALT decline in the all population $(p<0.001)$, the high-LDH subgroup $(p=0.04)$, and the low-LDH subgroup $(p<0.001)$ (Table 2).

\section{Tasit Improved Pt\% More Than Spt}

Transvenous high-dose corticosteroid was frequently administered to patients with ALF. We compared the survival rate, PT\% improvement, and ALT depression between the TASIT and systemic SPT groups to evaluate whether the administration route might affect the therapeutic effect (patients' background is shown in Supplemental Table 1). TASIT was more effective than SPT on prognosis and PT\% improvement as with the comparison between TASIT and no TASIT (Supplemental Table 2). Because of the small sample size, especially with the patients treated with SPT in the high-LDH subgroup, we could only detect statistical significance for PT\% improvement in the TASIT group in the all population. Multiple 
logistic regression analyses showed TASIT to be one of the significant factors related to the change in PT\% in the all population $(p<0.001)$ (Table 3$)$.

\section{Discussion}

In this study, we investigated the efficacy of TASIT for ALF treatment. We demonstrated that TASIT markedly increased PT\% in the all population and improved the survival rate in the high-LDH subgroup. Corticosteroids are known to inhibit an excessive immune response and inflammatory reaction, especially macrophage activation $[8,9]$. Several clinical studies have been carried out to evaluate the efficacy of administering corticosteroids to patients with ALF orally or transvenously, but the therapeutic effect is still controversial [10-13]. We speculated that corticosteroid administration via a peripheral vein could not achieve sufficient concentration in the liver due to the hepatic microcirculatory disturbance in ALF. Therefore, we investigated the efficacy of corticosteroid administration via a portal vein or hepatic artery in rats. We observed that the inflammatory cytokine expression, hepatocyte apoptosis, and the mortality rate were significantly lower in the portal vein injection group than in the tail vein injection group [27]. Similarly, TASIT was better than SPT in improving the prognosis and PT\% in this study. PT\% is a highly sensitive index for hepatic protein synthesis ability because it reflects the rapid turnover of coagulation factors. The improvement of PT\% by TASIT indicated that a sufficient corticosteroid concentration in the liver might suppress inflammatory cell activation, resulting in the recovery of hepatic synthetic ability in patients with ALF. We therefore hypothesize that TASIT could be a novel treatment option for patients with ALF.

The indications for corticosteroids in ALF have not yet been clarified, and some patients with ALF may be resistant to corticosteroid treatment. We stratified patients with ALF into high-LDH and low-LDH subgroups based on their serum ALT/LDH ratio. The high-LDH subgroup had serum ferritin elevation, microcirculatory disturbance, and subsequent intrahepatic hypoxia [20]. In this study, we analyzed the effectiveness of TASIT for ALF in high- and low-LDH subgroups and found that TASIT improved the prognosis and increased PT\% in ALF in the high-LDH, but not in the low-LDH, subgroup. Serum ferritin concentrations increase in acute hepatitis, and the source of ferritin is thought to be damaged hepatocytes [28]. However, some patients presented with a normal serum ferritin level despite a high serum ALT level. This strongly suggests that the increased ferritin might be derived from inflammatory cells, including activated macrophages [29,30]. Given that the higher serum ferritin level in the high-LDH subgroup might be due to advanced macrophage activation, it is reasonable that TASIT exerted a stronger therapeutic effect in the high-LDH subgroup than in the low-LDH subgroup. This study provides evidence that TASIT might improve the prognosis in ALF if it is administered to appropriate patients based on the ALT/LDH ratio.

We reported earlier that anticoagulant therapy ameliorated hepatic hypoxia and reduced liver damage in the ALI mouse model [31]. In the current study, the implementation ratio of anticoagulant therapy was higher in the high-LDH subgroup, but anticoagulant therapy only improved the serum ALT level in the lowLDH subgroup, and it had little impact on prognosis and PT\% improvement. Because we used several 
classes of anticoagulants, the variety of therapeutic targets and pharmacological actions could have affected the drug potency in patients with ALF. Further studies are needed to identify appropriate anticoagulant agents and the optimum combination of therapies, including anticoagulants and TASIT, to improve the prognosis of patients with ALF.

\section{Conclusions}

This study demonstrated that TASIT improved the prognosis and coagulation disorders in patients with ALF, especially in the subgroup with microcirculatory disturbance. Randomized controlled trials with a larger cohort are required to establish TASIT as a standard therapy for ALF.

\section{Abbreviations}

\section{ALF}

acute liver failure

LT

liver transplantation

ALI

acute liver injury

TASIT

transcatheter arterial steroid injection therapy

ALT

alanine aminotransferase

$\mathrm{LDH}$

lactate dehydrogenase

SPT

steroid pulse therapy

HAV

Hepatitis A virus

HBV

Hepatitis B virus

$\mathrm{AlH}$

autoimmune hepatitis

DILI

Drug-induced liver Injury

UK

unknown etiology

PT\%

prothrombin activity percentage

PT-INR 
prothrombin time-international normalized ratio

FDP

fibrin degradation products

AST

aspartate aminotransferase

Alb

albumin

TB

total bilirubin

Cre

creatinine

$\mathrm{NH}_{3}$

ammonia

MELD

model for end-stage liver disease

OR

odds ratio

$\mathrm{Cl}$

confidence interval.

\section{Declarations}

\section{Ethics approval and consent to participate}

This study was performed in accordance with the Declaration of Helsinki and approved by Ethics Committee of Kyushu University Hospital (No.27-377 and 28-432). Written informed consent for the treatment was obtained from the patient. Data analysis of his study was approved by Ethics Committee of Kyushu University Hospital and written informed consent was waived because of the retrospective design.

\section{Consent for publication}

Consent for publication were obtained from all patients.

\section{Availability of data and materials}

The dataset used and/or analyzed during the current study is available from the corresponding author on reasonable request.

\section{Competing interests}


The authors report no conflicts of interest. The authors are responsible for the content and writing of this article.

\section{Funding}

This work was supported in part by Takeda Science Foundation, Smoking Research Foundation, and JSPS KAKENHI (Grant Numbers: JP17K09430, JP18H05039, JP19H01054, JP19K17496, JP20K22877, JP2OH049).

\section{Author contributions}

A. Kuwano, M. Kohjima, T. Goya, M. Tanaka, M. Kato, and Y. Ogawa designed the study. A. Kuwano, T. Okui, M. Kohjima, M. Kurokawa, T. Aoyagi, M. Takahashi, K. Imoto, S. Tashiro, H. Suzuki, N. Fujita, Y. Ushijima, K. Ishigami, and S. Tokunaga collected data and assisted data analyses. T.Okui, M. Kohjima, M. Tanaka, S.Tokunaga, M. Kato, and Y. Ogawa contributed to analysis and interpretation of data. M. Kohjima, M. Tanaka, M. Kato and Y. Ogawa assisted in the preparation of the manuscript and critically reviewed the manuscript. All authors approved the final version of the manuscript and agreed to be accountable for all aspects of the work in ensuring that questions related to the accuracy or integrity of any part of the work.

\section{Acknowledgments}

The Authors would like to thank Enago (www.enago.jp) for the English language review.

\section{References}

1. Fujiwara K, Mochida S, Matsui A, Nakayama N, Nagoshi S, Toda G. Fulminant hepatitis and late onset hepatic failure in Japan. Hepatol Res. 2008;38(7):646-657.

2. Bernal W, Wendon J. Acute liver failure. N Engl J Med. 2013;369(26):2525-2534.

3. Starzl TE, Iwatsuki S, Van Thiel DH, Gartner JC, Zitelli BJ, Malatack JJ, Schade RR, Shaw BW Jr, Hakala TR, Rosenthal JT, Porter KA. Evolution of liver transplantation. Hepatology. 1982;2(5):614636.

4. Polson J, Lee WM. AASLD position paper: the management of acute liver failure. Hepatology. 2005;41(5):1179-1197.

5. Triantafyllou E, Woollard KJ, McPhail MJW, Antoniades CG, Possamai LA. The role of monocytes and macrophages in acute and acute-on-chronic liver failure. Front Immunol. 2018;9:2948.

6. Dong X, Liu J, Xu Y, Cao H. Role of macrophages in experimental liver injury and repair in mice. Exp Ther Med. 2019;17(5):3835-3847.

7. Møller HJ, Gronbaek H, Schiodt FV, Holland-Fischer P, Schilsky M, Munoz S, Hassanein T, Lee WM. Soluble CD163 from activated macrophages predicts mortality in acute liver failure. J Hepatol. 2007;47(5):671-676. 
8. Coutinho AE, Chapman KE. The anti-inflammatory and immunosuppressive effects of glucocorticoids, recent developments and mechanistic insights. Mol Cell Endocrinol. 2011;335(1):213.

9. Balow JE, Rosenthal AS. Glucocorticoid suppression of macrophage migration inhibitory factor. J Exp Med. 1973;137(4):1031-1041.

10. Zhao B, Zhang HY, Xie GJ, Liu HM, Chen Q, Li RF, You JP, Yang S, Mao Q, Zhang XQ. Evaluation of the efficacy of steroid therapy on acute liver failure. Exp Ther Med. 2016;12(5):3121-3129.

11. Czaja AJ. Corticosteroids or not in severe acute or fulminant autoimmune hepatitis: therapeutic brinksmanship and the point beyond salvation. Liver Transpl. 2007;13(7):953-955

12. Karkhanis J, Verna EC, Chang MS, Stravitz RT, Schilsky M, Lee WM, Brown RS Jr. Steroid use in acute liver failure. Hepatology. 2014;59(2):612-621.

13. Fujiwara K, Yasui S, Yonemitsu Y, Mikata R, Arai M, Kanda T, Imazeki F, Oda S, Yokosuka O. Efficacy of high-dose corticosteroid in the early stage of viral acute liver failure. Hepatol Res. 2014;44(5):491501.

14. Kotoh K, Enjoji M, Nakamuta M, Yoshimoto T, Kohjima M, Morizono S, Yamashita S, Horikawa $Y$, Yoshimitsu K, Tajima T, AsayamaY, Ishigami K, Hirakawa M. Arterial steroid injection therapy can inhibit the progression of severe acute hepatic failure toward fulminant liver failure. World $\mathrm{J}$ Gastroenterol. 2006;12(41):6678-6682.

15. Hirata K, Ogata I, Ohta Y, Fujiwara K. Hepatic sinusoidal cell destruction in the development of intravascular coagulation in acute liver failure of rats. J Pathol. 1989;158(2):157-165.

16. Mochida S, Arai M, Ohno A, Yamanobe F, Ishikawa K, Matsui A, Maruyama I, Kato H, Fujiwara K. Deranged blood coagulation equilibrium as a factor of massive liver necrosis following endotoxin administration in partially hepatectomized rats. Hepatology. 1999;29(5):1532-1540.

17. Vollmar B, Glasz J, Leiderer R, Post S, Menger MD. Hepatic microcirculatory perfusion failure is a determinant of liver dysfunction in warm ischemia-reperfusion. Am J Pathol. 1994;145(6):14211431.

18. Palmes D, Skawran S, Stratmann U, Armann B, Minin E, Herbst H, Spiegel HU. Amelioration of microcirculatory damage by an endothelin $A$ receptor antagonist in a rat model of reversible acute liver failure. J Hepatol. 2005;42(3):350-357.

19. Hillenbrand P, Parbhoo SP, Jedrychowski A, Sherlock S. Significance of intravascular coagulation and fibrinolysis in acute hepatic failure. Gut. 1974;15(2):83-88.

20. Kuwano A, Kurokawa M, Kohjima M, Imoto K, Tashiro S, Suzuki H, Tanaka M, Okada S, Kato M, Ogawa Y. Microcirculatory disturbance in acute liver injury. Exp Ther Med. 2021;21(6):596.

21. Mochida S, Takikawa Y, Nakayama N, Oketani M, Naiki T, Yamagishi Y, Ichida T, Tsubouchi H. Diagnostic criteria of acute liver failure: a report by the intractable hepato-biliary diseases study group of Japan. Hepatol Res. 2011;41(9):805-812.

22. Davenport A, Will EJ, Davison AM. Effect of renal replacement therapy on patients with combined acute renal and fulminant hepatic failure. Kidney Int Suppl. 1993;41:S245-S251. 
23. Robert $A$, Chazouillères 0 . Prothrombin time in liver failure: time, ratio, activity percentage, or international normalized ratio? Hepatology. 1996;24(6):1392-1394.

24. Pauwels A, Mostefa-Kara N, Florent C, Lévy VG. Emergency liver transplantation for acute liver failure. Evaluation of London and Clichy criteria. J Hepatol. 1993;17(1):124-127.

25. 28. Canbay A, Chen SY, Gieseler RK, Malago M, Karliova M, Gerken G, Broelsch CE, Treichel U. Overweight patients are more susceptible for acute liver failure. Hepatogastroenterology. 2005;52(65):1516-1520.

26. Rueff B, Benhamou JP. Acute hepatic necrosis and fulminant hepatic failure. Gut. 1973;14(10):805815.

27. Higuchi N, Kato M, Kotoh K, Kohjima M, Aishima S, Nakamuta M, Fukui Y, Takayanagi R, Enjoji M. Methylprednisolone injection via the portal vein suppresses inflammation in acute liver failure induced in rats by lipopolysaccharide and d-galactosamine. Liver Int. 2007;27(10):1342-1348.

28. Bhagat Cl, Fletcher S, Joseph J, Beilby JP. Plasma ferritin in acute hepatocellular damage. Clin Chem. 2000;46(6 Pt 1):885-886.

29. Kotoh K, Ueda A, Tanaka M, Miyazaki M, Kato M, Kohjima M, Enjoji M, Nakamuta M, Takayanagi R. A high prevalence of extreme hyperferritinemia in acute hepatitis patients. Hepat Med. 2009;1:1-8.

30. Matsui A, Mochida S, Ohno A, Nagoshi S, Hirose T, Fujiwara K. Plasma osteopontin levels in patients with fulminant hepatitis. Hepatol Res. 2004;29(4):202-206.

31. Kuwano A, Kohjima M, Suzuki H, Yamasaki A, Ohashi T, Imoto K, Kurokawa M, Morita Y, Kato M, Ogawa Y. Recombinant human soluble thrombomodulin ameliorates acetaminophen-induced liver toxicity in mice. Exp Ther Med. 2019;18(2):1323-1330.

\section{Tables}

Table 1-3 is available in the Supplemental Files section.

\section{Figures}


Figure 1

All population
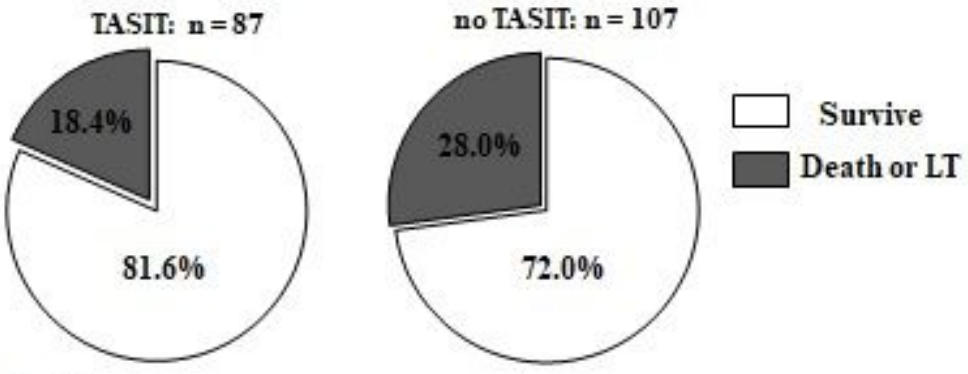

high-LDH subgroup
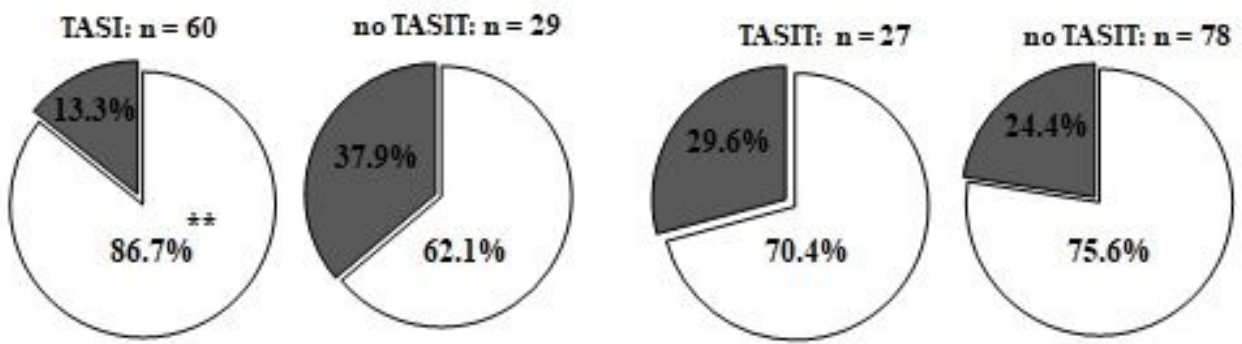

\section{Figure 1}

The prognosis of patients with ALF.

${ }^{*} p<0.05,{ }^{* *} p<0.01$ compared with no TASIT group.

TASIT: Transcatheter arterial steroid injection therapy, LT: liver transplantation, LDH: lactate dehydrogenase. 
Figure 2
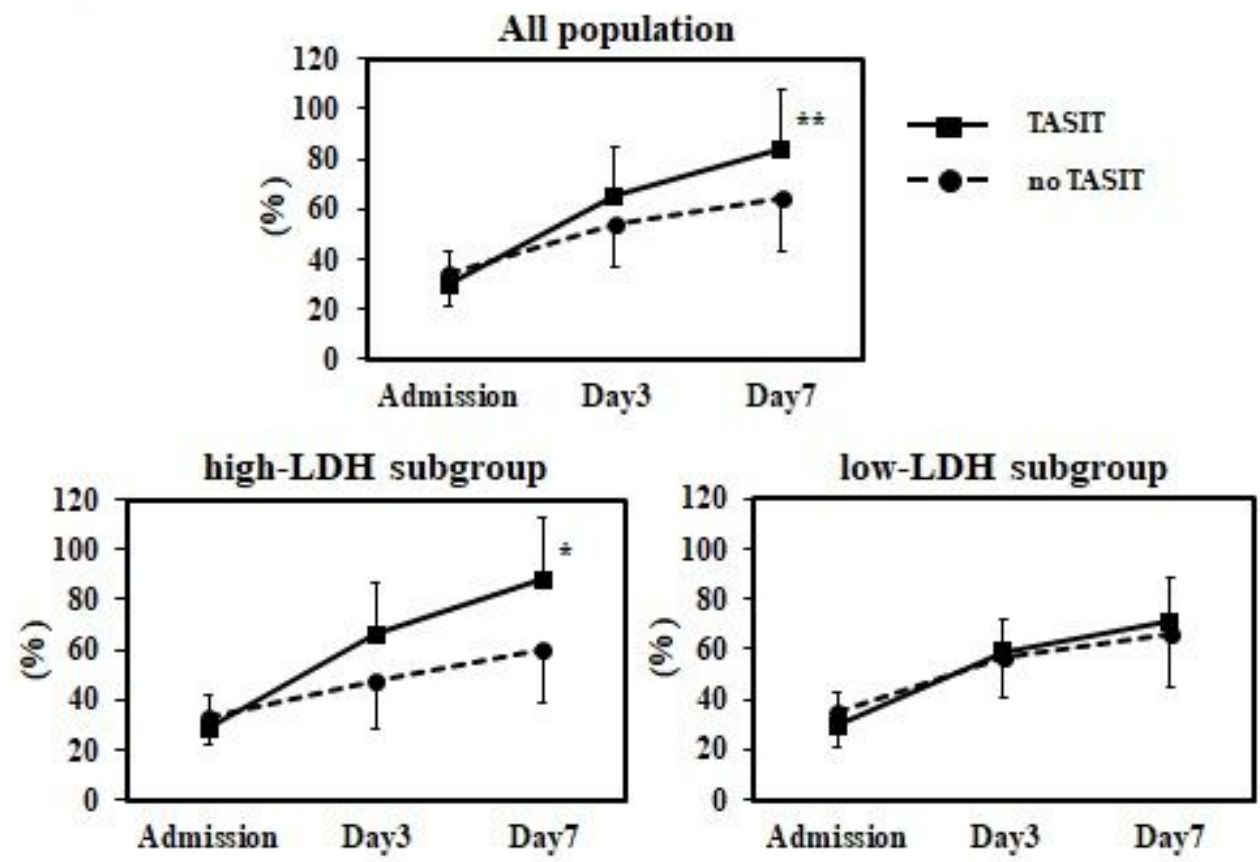

Figure 2

The change in PT\% value in patients with ALF.

${ }^{*} \mathrm{p}<0.05,{ }^{* *} \mathrm{p}<0.01$ compared with no TASIT group.

TASIT: Transcatheter arterial steroid injection therapy, LDH: lactate dehydrogenase, PT\%: prothrombin activity percentage. 
Figure 3
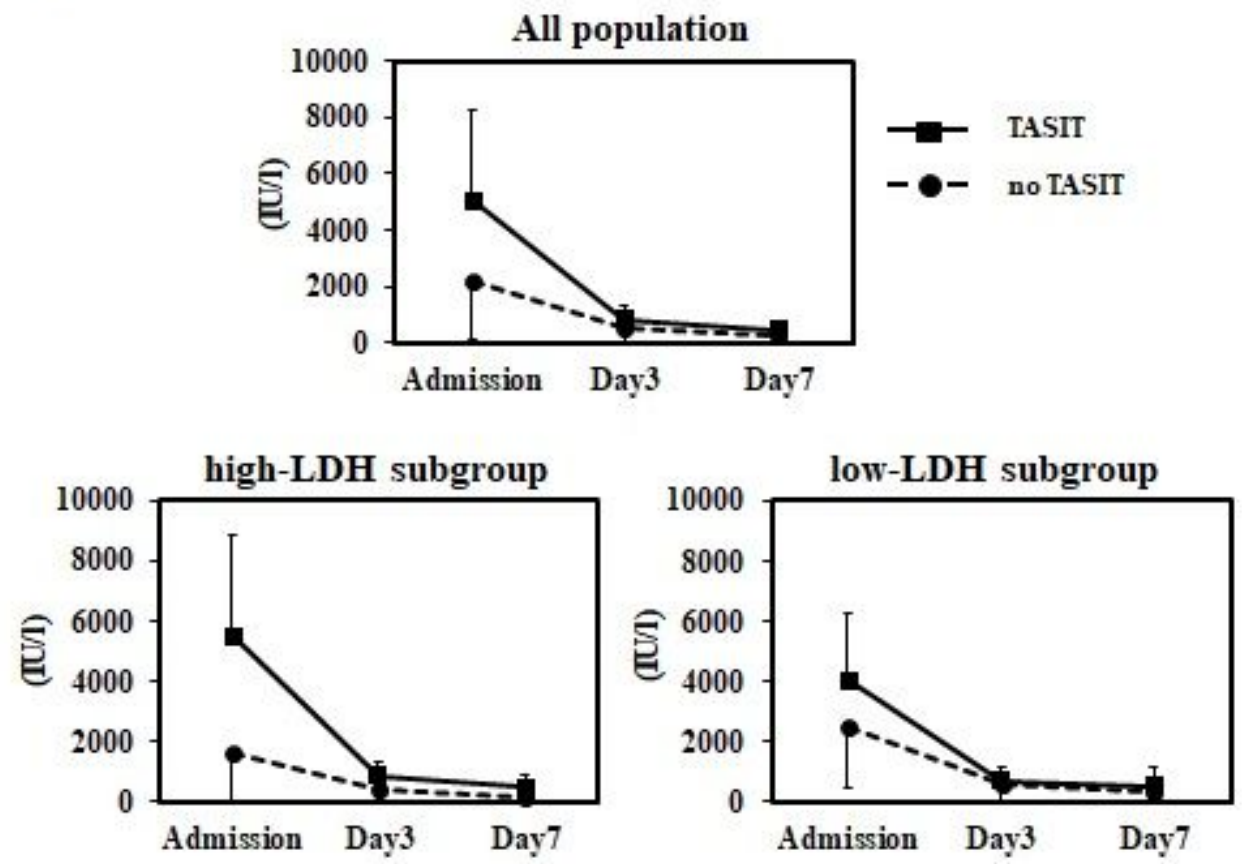

Figure 3

The change in serum ALT value in patients with ALF.

${ }^{*} \mathrm{p}<0.05,{ }^{* \star} \mathrm{p}<0.01$ compared with no TASIT group.

TASIT: Transcatheter arterial steroid injection therapy, LDH: lactate dehydrogenase, ALT: alanine aminotransferase.

\section{Supplementary Files}

This is a list of supplementary files associated with this preprint. Click to download.

- TASITTablesBMC.pptx

- LegendsSupplementallyTablesBMC.docx

- TASITsuppl.TablesBMC.pptx 